\title{
Über Verbindungen des zweiwertigen Kobalts mit Brenzkatechin.
}

Von R. F. Weinlaand und Anna Dötringer.

Daß das Brenzkatechin mit Metallen überaus beständige komplexe Anionen zu bilden vermag, wurde zuerst beim dreiwertigen Eisen beobachtet. ${ }^{1}$ Es gibt zwei Reihen solcher FerriBrenzkatechin-Verbindungen, nämlich einmal Salze des tiefroten Tribrenzkatechin-ferri-anions und sondann Salze des violetten Dibrenzkatechin-ferri-anions:

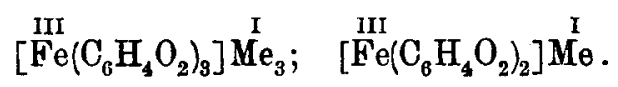

Die ersteren entstehen stets, wenn Brenzkatechin in alkalischer Lösung mit dreiwertigem Eisen zusammentrifft, sie bilden sich aber auch mit zweiwertigem Eisen, indem dieses durch den Sauerstoff der Luft rasch oxydiert wird. ${ }^{2}$ Die Salze der violetten Reihe bilden sich in neutraler oder essigsaurer Lösung; hier wurde auch die freie Dibrenzkatechin-ferri-Säure aufgefunden:

$$
\left[\mathrm{Fe}\left(\mathrm{C}_{6} \mathrm{H}_{4} \mathrm{O}_{2}\right)_{2}\right] \mathrm{H} .2 \mathrm{H}_{2} \mathrm{O} \text {. }
$$

Die violetten Salze gehen unter der Einwirkung von Alkalien in die roten über, wobei sich der dritte Teil des Fisens als Ferrihydroxyd abscheidet:

$$
3\left[\mathrm{Fe}\left(\mathrm{C}_{6} \mathrm{H}_{4} \mathrm{O}_{2}\right)_{2}\right] \mathrm{K}+3 \mathrm{KOH}=2\left[\mathrm{Fe}\left(\mathrm{C}_{6} \mathrm{H}_{4} \mathrm{O}_{2}\right)_{3}\right] \mathrm{K}_{3}+\mathrm{Fe}(\mathrm{OH})_{3} \text { ' }
$$

Diese zwei Reihen von Brenzkatechinanionen wurden sodann auch bei dem Leichtmetall Aluminium ${ }^{3}$ aufgefunden.

Daß zweiwertige Metalle mit Brenzkatechin ebenfalls alkalibeständige Komplexe geben, ist leicht durch Reagens-

1 Weinland u. K. Binder, Ber. deutsch. chem. Ges. 45 (1912), 148, 1113.

${ }^{2}$ Die Aufnahme des Sauerstoffs durch eine alkalische FerrosulfatBrenzkatechinlösung erfolgt mit solcher Geschwindigkeit und mit so intensiver Rotfärbung, daß jene Lösung ein außerordentlich scharfes Reagens für Sauerstoff ist. Siehe Binder u. Wetnland, Ber. dieutsch. chem. Ges. 46 (1913), 255.

${ }^{3}$ Weinland u. W. Denzei, Ber. deutsch. chem. Ges. 47 (1914), 737, 2753 
rohrversuche festzustellen: Fügt man zu einer mit Brenzkatechin versetzten Lösung eines Kobalto-, Nickel-, Mangano-oder Ferrosalzes Alkalıhydroxyd, so wird kein Metallhydroxyd gefällt, das Brenzkatechin maskiert also das Metall. Eine Untersuchung darüber, um welche Komplexe es sich beim zweiwertigen Kobalt handelt, ergab das Folgende.

\section{A. Darstellung der Verbindungen.}

Wässerige Lösungen der gewöhnlichen Kobaltsalze als solche reagieren nicht mit Brenzkatechin, es tritt weder eine Farbänderung, noch ein Niederschlag auf. ${ }^{1}$ Fügt man jedoch Basen hinzu, so erhält man Verbindungen des zweiwertigen Kobalts teils mit Brenzkatechin allein, teils mit Brenzkatechin und der betreffenden Base. Die Basen, die wir verwendeten, waren Ammoniak, Pyridin und Kaliumhydroxyd.

Aus Lösungen von Kobaltsulfat, die mit verschiedenen Mengen Brenzkatechin und Ammoniak in wechselnder Verdünnung versetzt wurden, konnten die folgenden Verbindungen erhalten werden. Der Übersichtlichkeit halber sind sie sogleich mit ihren Konstitutions-, bzw. stöchiometrisch anschaulichen Formeln angeführt. Die Erörterung der Konstitution wird weiter unten folgen (S. 228).

I. $\left[\mathrm{Co}\left(\mathrm{C}_{6} \mathrm{H}_{4} \mathrm{O}_{2}\right)_{2}\right] \mathrm{H}_{2} \cdot 5^{1 / 1} \mathrm{H}_{2} \mathrm{O}$, hellrote, schief abgeschnittene, vier- oder sechsseitige Säulchen.

II. $\left[\mathrm{Co}\left(\mathrm{C}_{6} \mathrm{H}_{4} \mathrm{O}_{2}\right)_{2}\right]\left(\mathrm{NH}_{4}\right)_{2}$, hellrote, gerado abgeschnittene, vierseitige Säulchen.

III. $3\left[\mathrm{Co}\left(\mathrm{C}_{6} \mathrm{H}_{4} \mathrm{O}_{2}\right)_{2}\right]\left(\mathrm{NH}_{4}\right)+\left[\mathrm{Co}_{(}\left(\mathrm{C}_{6} \mathrm{H}_{4} \mathrm{O}_{2}\right)_{2}\right]\left(\mathrm{NH}_{4}\right)_{2}+\mathrm{H}_{2} \mathrm{O}$, rote, eisblumenartige Aggregate.

IV. $\left[\mathrm{Co}\left(\mathrm{C}_{6} \mathrm{H}_{4} \mathrm{O}_{2}\right)_{3}\right]\left(\mathrm{NH}_{4}\right)_{4}+\mathrm{C}_{6} \mathrm{H}_{4}(\mathrm{OH})_{2}$, sehr blabrote, dünne Täfelchen.

V. $\mathrm{Co}_{3}\left(\mathrm{C}_{6} \mathrm{H}_{4} \mathrm{O}_{2}\right)_{2}(\mathrm{OH})_{2} \cdot 6 \mathrm{H}_{2} \mathrm{O}$, rosarotes Pulver.

Betreffs der präparativen Einzelheiten verweisen wir auf den experimentellen Teil, S. 284 ff., im allgemeinen bemerken wir hierüber folgendes:

Welche der angeführten Verbindungen man aus der betreffenden Lösung bekommt, ist weniger vom Mengenverhältnis des Kobalt-

1 Kobaltacetat gibt beim Kochen mit Brenzkatechin einen blaßroten, pulverigen Niederschlag, den wir bis jetzt nicht weiter untersucht haben. 
salzes und des Brenzkatechins als von der Menge des zugesetzten A mmoniaks abhängig.

Auf Zusatz von Ammoniak zu Lösungen bestimmter Konzentration von Kobaltsulfat, die mit Brenzkatechin versetzt sind, scheidet sich stets zunächst ein blaßroter, pulverförmiger Niederschlag aus, der, wenn die Menge des Ammoniaks klein ist (auf ein Mol. Kobaltsalz etwa ein Mol. Ammoniak), unverändert in der Flüssigkeit bleibt. $\mathrm{Er}$ ist ammoniakfrei und stellt ein basisches Kobaltbrenzkatechinat von stets gleicher Zusammensetzung vor in der obigen Liste $\mathrm{Nr}$. V. Ist die Menge des zugesetzten Ammoniaks gröBer (auf ein Mol. Kobaltsalz zwei bis fünf Mol. Ammoniak), so verwandelt sich das basische Kobaltbrenzkatechinat in der Fällungsflüssigkeit mehr oder weniger raseh in die Verbindung I bzw. III. Die erstere ist noch ammoniakfrei, die zweite ist ammoniakhaltig. Beide enthalten auf ein Atom Kobalt zwei Brenzkatechinreste. Fügt man zu einem Mol. Kobaltsalz etwa zehn Mol. Ammoniak, so löst sich das zuerst ausfallende basische Brenzkatechinat Nr. V sogleich wieder auf. Aus dieser Lösung scheidet sich je nach der Konzentration entweder die Verbindung II oder die Verbindung IV aus.

Bei geringerer Konzentration erhält man die Verbindung II in wenig guter Ausbeute, aber sehr schön kristallisiert. Sie erleidet in der Mutterlauge keinerlei Veränderung. Aus stark konzentrierter Lösung scheidet sich die Verbindung IV sofort in reichlicher Menge aus; aber sie ist in der Fällungsflüssigkeit nicht beständig, sondern verwandelt sich darin ziemlich rasch teilweise in die Verbindung II. Nimmt man dagegen sehr viel Ammoniak (auf ein Mol. Kobaltsalz etwa $30 \mathrm{Mol}$. Ammoniak) und arbeitet in möglichst konzentrierter Lösung, so erhält man ausschließlich die Verbindung IV und sie erleidet in der Mutterlauge keine Veränderung mehr. ${ }^{1}$

Im Gegensatz zum Ammoniak gibt das Pyri din, in welcher Menge es auch zu einer Lösung von Kobaltsulfat und Brenzkatechin hinzugefügt wird, stets nur eine und dieselbe Verbindung der Zusammensetzung

$$
\text { VI. } 4\left[\mathrm{Co}\left(\mathrm{C}_{6} \mathrm{H}_{4} \mathrm{O}_{2}\right)_{2}\right] \mathrm{H}_{2} \cdot\left(\mathrm{C}_{5} \mathrm{H}_{5} \mathrm{~N}\right)_{2}+\mathrm{C}_{6} \mathrm{H}_{4}(\mathrm{OH})_{2}
$$

als schwerlöslichen, fleischfarbenen, sehr klein kristallinischen Niederschlag. Auch durch weitgehenden Wechsel in der Menge des

1 Es sei noch bemerkt, daß alle ammoniakalischen, brenzkatechinhaltigen Kobaltsalzlösungen sich, wie alkalische Brenzkatechinlösungen überhaupt, an der Luft grün und schließlich schwarz färben.

Z. anorg. u, allg. Chem. Bd. 102. 
Brenzkatechins erhält man keine andere Verbindung. Man kann Lieraus schließen, daß das obige Pyridinsalz schwerer löslich ist als die basenfreien Verbindungen Nr. V und Nr. I (s. S. 224), und ferner, daß es unter den möglichen Pyridinverbindungen die weitaus schwerlöslichste ist.

Fügt man Kaliumhydroxyd zu den mit Brenzkatechin versetzten Lösungen von Kobaltsulfat, bzw. -acetat ${ }^{1}$, so bekommt man, wie beim Ammoniak, auf Zusatz kleiner Mengen das basische Kobaltbrenzkatechinat Nr. V. Nimmt man mehr Kaliumhydroxyd (Co: $\mathrm{KOH}=1: 2$ ), so entsteht, ebenfalls wie beim Ammoniak, die Verbindung Nr. I. Bei noch weiterer Vermehrung des Kaliumhydroxyds ( $\mathrm{Co}: \mathrm{KOH}=1: 3$ ) erhält man dagegen die alkalifreje Verbindung Nr. I nicht mehr allein, sondern vermischt mit einem sich in violetten Kristallen ausscheidenden Salz der Zusammensetzung

$$
\text { VII. }\left[\mathrm{Co}\left(\mathrm{C}_{6} \mathrm{H}_{4} \mathrm{O}_{2}\right)_{2}\right]_{\mathrm{H}}^{\mathrm{K}} \cdot 2 \mathrm{H}_{2} \mathrm{O} \text {. }
$$

Letztere Verbindung allein bekommt man durch abermalige Steigerung der Menge des zugesetzten Kaliumhydroxyds (1 Co: $4 \mathrm{KOH}$ ), wobei man außerdem noch die Menge des Brenzkatechins erhöht und konzentrierter arbeitet. Setzt man endlich Kaliumhydroxyd in außerordentlich großer Menge zu (1 Co: $40 \mathrm{KOH})$, so erhält man rote, gerade abgeschnittene Säulchen von folgender Zusammensetzung:

$$
\text { VIII. }\left[\mathrm{Co}\left(\mathrm{C}_{6} \mathrm{H}_{4} \mathrm{O}_{2}\right)_{3}\right] \mathrm{K}_{4} \cdot 4 \mathrm{H}_{2} \mathrm{O} \text {. }
$$

$\mathrm{DaB}$ man aus so stark alkalischen Lösungen, wie sie zur Darstellung des Salzes Nr. VIII notwendig sind, sehr schön kristallisierte Verbindungen erhält, wurde zum erstenmal beim Kochen des obigen Pyridinsalzes (Nr. VI, S. 225) mit Kalilauge beobachtet.

Bei den alkalihydroxydhaltigen Kobaltosalz-Brenzkatechinlösungen ist es eigentümlich, $\mathrm{da} B$ die alkaliärmeren sich wie die ammoniakalischen an der Luft dunkelgrün bis schwarz färben, dagegen die sehr alkalireichen ihre ursprünglich blaue bzw. rote Farbe behalten, in ihnen das Brenzkatechin also nicht oxydiert wird. ${ }^{2}$

1 Bei der Darstellung der leicht löslichen Alkaliverbindungen mußteKobaltacetat verwendet werden, da bei der Anwendung von Sulfat sich das entstehende Alkalisulfat in den konzentrierten Lösungen ausscheidet und die Körper verunreinigt, während das Alkaliacetat in Lösung bleibt.

2 Diese eigentümliche Beobachtung veranlaßte uns, zu untersuchen, wie sich Lösungen von Alkalihydroxyden verhalten, in denen Brenzkatechin allein gelöst wurde. Auch hierbei fanden wir, daß sich die schwach alkalischen Iösungen rascher und stärker dunkel färben als die stark alkalischen. Das Brenzkatechin oxydiert sich also in konzentrierten Laugen viel langsamer als in verdünnten. 


\section{B. Eigenschaften der Verbindungen.}

Für alle angeführten Verbindungen, mit Ausnahme des Pyridinsalzes, ist charakteristisch, $\mathrm{da} B$ sie sich, nachdem sie von der Mutterlauge getrennt, gewaschen und durch Absaugen möglichst von Flüssigkeit befreit sind, verändern, indem sie, an der Luft oder über Chlorcalcium, ihre ursprünglich rote bzw. violette Farbe durch oberflächliche Oxydation verlieren und sich mehr oder weniger rasch dunkel färben.

Was die Farbe an sich betrifft, so ist sie bei der Mehrzahl nicht wesentlich verschieden von dem bekannten Rot der gewöhnlichen, wasserhaltigen Kobaltosalze, nur ein Kaliumsalz, das Salz Nr. VII, ist rot- bis blauviolett, und das Pyridinsalz ist fleischfarben.

Die Löslichkeit der Verbindungen in Wasser ist sehr verschieden; so sind z. B. der ammoniakfreie Körper Nr. I, das ammoniakfreie, basische Salz Nr. V und der Pyridinkörper Nr. VI schwer löslich, während die alkalihaltigen Salze Nr. VII und VIII und der Ammoniumkörper Nr. IV leicht löslich sind. Die alkalifreien Verbindungen Nr. I und V und der Pyridinkörper lösen sich mit blaBroter Farbe, die alkalireiche Verbindung Nr. VIII mit roter Farbe. Sowohl die blaßrote als die rote Lösung färben sich infolge von Oxydation rasch dunkel. Alle übrigen Verbindungen lösen sich in Wasser sogleich mit grüner Farbe. Eine Umkristallisation aus Wasser ist bei keiner der Verbindungen möglich.

Gegen Alkalihydroxyde sind die Verbindungen beständig. Sie lösen sich nämlich in Alkalilösungen mit violettroter Farbe, welche Farbe die Lösung auch beim Kochen beibehält. Fs wird in keinem Falle Kobaltohydroxyd gefällt. Der Pyridin- und die Ammoniumkörper werden durch Alkalien in Alkalisalze verwandelt.

Schwefelammon dagegen fällt sofort Schwefelkobalt, und ferner bildet sich beim Kochen der. Verbindungen mit einer Lösung von Kaliumcyanid Kaliumkobalticyanid, was daraus hervorgeht, daß die mit Kaliumcyanid erwärmten Lösungen durch Schwefelammon nicht mehr gefällt werden. - Dinatriumphosphat fällt aus den alkalischen Lösungen, auch beim Kochen, kein Kobalt. Dagegen bildet sich beim Kochen der festen Verbindungen mit Dinatriumphosphatlösung Kabaltphosphat.

Aus diesem Verhalten der Verbindungen gegen Schwefelammon, Kaliumeyanid und Dinatriumphosphat geht hervor, $\mathrm{da} B$ die ihnen zugrunde liegenden Komplexe, nämlich Brenzkatechinatokobalto-anionen (s. unten S. 228), wesentlich weniger beständig sind, 
als das rote Tribrenzkatechinato-ferrianion (S. 223), welches von den genannten Reagenzien auch bei langem Kochen nur wenig angegriffen wird.

In Säuren lösen sich die Verbindungen sogl, ich unter Zer. setzung in Kobaltosalz und freies Brenzkatechin mit blaBroter Farbe; letzteres kann man ausäthern.

Fügt man zu diesen sauren Lösungen Kaliumjodid ${ }^{1}$, so wird kein Jod oder nur sehr wenig frei gemacht. Daraus geht hervor, da $B$ die Verbindungen zweiwertiges Kobalt enthalten.

\section{Konstitution der Verbindangen.}

Die im obigen angeführten und zum Teil bereits in Konstitutions. formeln geschriebenen alkali- bzw. ammoniakbaltigen Verbindungen können der Analyse nach rein stöchiometrisch als Doppelverbindungen von Kobaltbrenzkatechinat mit Alkali- bzw. Ammoniumbrenzkatechinat formuliert werden, z. B. das Ammoniumsalz Nr. II und das Kaliumsalz Nr. VIII folgendermaßen:

$$
\begin{aligned}
& \text { II. } \mathrm{Co}\langle\stackrel{\mathrm{O}}{\mathrm{O}}\rangle \mathrm{C}_{6} \mathrm{H}_{4}+\mathrm{C}_{6} \mathrm{H}_{4}\left(\mathrm{ONH}_{4}\right)_{2} \text {; } \\
& \text { VIII. } \mathrm{Co}_{0}\left\langle{ }_{\mathrm{O}}^{\mathrm{O}}\right\rangle \mathrm{C}_{6} \mathrm{H}_{4}+2 \mathrm{C}_{6} \mathrm{H}_{4}(\mathrm{OK})_{2}+4 \mathrm{H}_{2} \mathrm{O} \text {. }
\end{aligned}
$$

Für ihre Konstitution jedoch ist entscheidend, daß sie durchweg gegen Alkalien beständig sind. Daher müssen ihnen komplexe Kobalt-brenzkatechin-anionen zugrunde liegen. Dies steht damit in Übereinstimmung, daß das Brenzkatechin auch mit anderen Metallen, so mit dem dreiwertigen Eisen und Aluminium, sehr beständige komplexe Anionen bildet (S. 223), und ferner damit, daß das zweiwertige Kobalt seinerseits fähig ist, mit gewissen organischen Säuren, wie der Oxalsäure, auch komplexe Anionen zu bilden. Man kennt ein Di- und ein Tri-oxalato-kobalto-anion:2

$$
\left[\mathrm{Co}\left(\mathrm{C}_{2} \mathrm{O}_{4}\right)_{2}\right] \mathrm{K}_{2} \cdot 6 \mathrm{H}_{2} \mathrm{O} ;\left[\mathrm{Co}\left(\mathrm{C}_{2} \mathrm{O}_{4}\right)_{8}\right]\left(\mathrm{NH}_{4}\right)_{4} \cdot 6 \mathrm{H}_{2} \mathrm{O} \text {. }
$$

Jemgemä $B$ sind in dem obigen Kaliumsalz Nr. VIII die drei

1 Diese Versuche sind mit den frisch dargestellten Salzen, unmittelbar nach dem Auswaschen, ehe sie sich an der Luft dunkel gefärbt haben, auszuführen. Ältere, dunkel gefärbte Präparate scheiden etwas mehr Jod aus. Es sei noch hinzugefügt, daß alkalische (kobaltfreie) Brenzkatechinlösungen, die sich an der Luft grün-schwarz gefärbt haben, aus angesäuerter Kaliumjodidlösung etwas Jod in Freiheit setzen.

${ }^{2}$ Rammmesbera, Beitstioin, Handb. d. organ. Chem. 1, 644; Fr. Ephraim Ber. deutsch. chem. Ges. 42 (1909), 3850. 
Brenzkatechinreste mit dem Kobalt zu einem vierbasischen Anion vereinigt und bilden die vier Kaliumatome das Kation:

$$
\text { VIII. }\left[\mathrm{Co}\left(\mathrm{C}_{6} \mathrm{H}_{4} \mathrm{O}_{2}\right)_{6}\right] \mathrm{K}_{4} \cdot 4 \mathrm{H}_{2} \mathrm{O} \text {. }
$$

Es ist als Kalium-tribrenzkatechinato-kobaltoat zu bezeichnen. Damit steht dieses Salz in Parallele zu dem roten Kaliumtribrenzkatechinato-ferriat (S. 228):

$$
\left[\mathrm{Fe}\left({ }_{3} \mathrm{O} \mathrm{C}_{3} \mathrm{H}_{4}\right)_{3}\right] \mathrm{K}_{3} \cdot 2 \mathrm{H}_{2} \mathrm{O} \text {. }
$$

Die Koordinationszahl des Zentralatoms ist in beiden Anionen gleich sechs. ${ }^{1}$ Ferner bilden sich beide Verbindungen in stark alkalischer Lösung und werden durch Säuren zersetzt. Ein großer Unterschied besteht allerdings zwischen diesen Brenzkatechinato-ferri- und -kobaltoanionen, und zwar in Bezug auf die Farbe. Während bei dem Ferri-anion eine neue typische Farbe auftritt, nämlich ein tiefes Rot, das so intensiv ist, daß es als Reaktion auf Eisen sowohl als auf Brenzkatechin und Sauerstoff (s. o. S. 223) benützt werden kann, zeigt das Kobalto-anion eine wenig intensive, von dem Rot der gewöhnlichen, wasserhaltigen Kobaltosalze kaum abweichende Farbe.

Ein derartiges komplexes Brenzkatechinato-kobalto-anion muß nun auch das oben (S. 228) als Doppelverbindung aufgeführte Ammoniumsalz Nr. II besitzen. Es ist jedoch insofern anders zusammengesetzt, als es auf ein Atom Kobalt nur zwei Brenzkatechinreste enthält und infolgedessen zweibasisch ist:

$$
\text { II. } \mathrm{Co}\left(\left\langle\mathrm{O}_{\mathrm{O}}^{\mathrm{O}} \mathrm{C}_{6} \mathrm{H}_{4}\right)_{2}\right]\left(\mathrm{NH}_{4}\right)_{2} \text {. }
$$

Ammonium-dibrenakatechinato-kobaltost.

In diesem Anion hat das Kobaltatom die Koordinationszahl 4, während diese im obigen Kaliumsalz Nr. VIII 6 beträgt; die Koordinationszahl kann also bei derartigen Kobaltokomplexen schwanken.

Die Dibrenzkatechinokobaltoate können den oben (S. 223) erwähnten, tiefvioletten Dibrenzkatechinato-ferrisalzen an die Seite gestellt werden, so z. B. dem Ammoniumsalz:

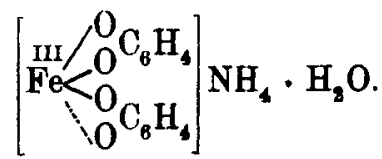

1 In derartigen Anionen äußert das Zentralatom Nebenvalenzen gegen einige der Sauerstoffatome der Säurereste, wie daraus hervorgeht, da $B$ die blauen Trioxala tochromiate sich in Spiegelbildisomere haben spalten lassen, siehe WrankR, Ber. deutsch. chem. Ges. 45 (1902), 3061. 
Beirn Vergleich der Farben der Dibrenzkatechinato- und Tribrenzkatechinato-kobalto-anionen untereinander und der entsprechenden Ferri-anionen treten jedoch große Unterschiede zutage. Bei den letzteren ist das eine tiefrot, das andere aber violett, die Farbverschiedenheit daher ganz bedeutend. Es tritt somit mit der Änderung der Zusammensetzung des Komplexes eine völlig neue Farbe auf. Dagegen ist das Tribrenzkatechinato-anion des Kobalts vom Dibrenzkatechinato-anion in der Farbe wenig verschieden, diese somit für das betreffende Anion nicht charakteristisch.

Dasselbe Dibrenzkatechinato-kobalto-anion enthalten sodann noch vier der•von uns dargestellten Verbindungen, nämlich Nr. I, III, VI und VII.

Das Ammoniumsalz Nr. III würde, als Doppelsalz geschrieben, folgende Formel haben:

$$
4 \mathrm{C}_{6} \mathrm{H}_{4} \mathrm{O}_{2} \mathrm{Co}+3 \mathrm{C}_{6} \mathrm{H}_{4}(\mathrm{OH})\left(\mathrm{ONH}_{4}\right)+\mathrm{C}_{6} \mathrm{H}_{4}\left(\mathrm{ONH}_{4}\right)_{2}+\mathrm{H}_{2} \mathrm{O} \text {. }
$$

Man sieht, daß auf 4 Kobaltatome im ganzen 8 Brenzkatechinreste kommen. Hieraus kann ohne weiteres geschlossen werden, daB auch diesem Salze das Dibrenzkatechinato-kobalto-anion zugrunde liegt. Da auf die 4 zweibasischen Dibrenzkatechinato-kobaltoanionen nur 5 Ammoniumgruppen kommen, handelt es sich um ein teilweise saures Salz, und zwar um eine Doppelverbindung von drei Molekülen Monoammonium-dibrenzkatechinato-kobaltoat mit einem Molekül Diammonium-dibrenzkatechinato-kobaltoat:

$$
\text { III. } 3\left[\mathrm{Co}\left(\mathrm{C}_{6} \mathrm{H}_{4} \mathrm{O}_{2}\right)_{2}\right] \underset{\mathrm{H}}{\mathrm{NH}_{4}} \cdot\left[\mathrm{Co}\left(\mathrm{C}_{6} \mathrm{H}_{4} \mathrm{O}_{2}\right)_{2}\right]\left(\mathrm{NH}_{4}\right)_{2} \cdot \mathrm{H}_{2} \mathrm{O} \text {. }
$$

Derartigen eigentümlichen Doppelverbindungen begegnete man auch bei den Ammonium- und Kaliumsalzen des Dibrenzkatechinatoferri-anions ${ }^{1}$, sowie bei den Brenzkatechinato-aluminiumsalzen. ${ }^{2}$

Das Kaliumsalz Nr. VII enthält auf ein Kobaltatom wiederum zwei Brenzkatechinreste, aber nur ein Kaliumatom. Nach dem Vorhergehenden kann man es ohne Bedenken als saures Kaliumsalz des obigen Dibrenzkatechinato-kobalto-anions ansehen:

$$
\text { VII. } \left.\left[\mathrm{Co}\left(\mathrm{C}_{6} \mathrm{H}_{6} \mathrm{O}_{2}\right)_{2}\right]\right]_{\text {Monokalium-dibrenzkatechloato-kobaltoat. }}^{\mathrm{K}} \cdot 2 \mathrm{H}_{2} \mathrm{O} .
$$

Es stellt die einfachste Form eines sauren Salzes jenes Anions vor. Bei diesem Salze fällt die rot-bzw. blauviolette Farbe auf,

1 Whinland u. K. Binder. Ber. deutsch. chem. Ges. 45 (1912), 1113.

2 Weinland u. W. Dingei, Ber. deutsch. chem. Ges. 47 (1914), 2753. 
insofern die anderen Salze mit demselben Anion das Rot der gewöhnlichen wasserhaltigen Kobaltosalze zeigen. Da das Salz zwei Mol. Wasser enthält, könnte man daran denken, daß diese zum komplexen Anion gehören. Dann würde es sich um ein neues Anion:

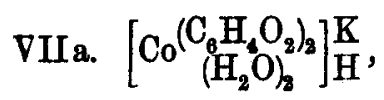

ein Diaquo-dibrenzkatechinato-kobalto-anion, handeln, das möglicherweise die violette Farbe besäße. Indessen kommt es auch sonst vor, dab Salze ein und desselben Komplexes in der Farbe schwanken, so werden z. B. die Salze des Hexamminkobalti-kations (Luteosalze) mit farblosen Säuren teils als rein gelb (wie Musivgold), teils als braungelb, teils als weinrot oder bräunlich orangerot beschrieben.

Da die Koordinationszahl des Kobalts in den Brenzkatechinanionen sowohl vier (wie in der Formel VII) als auch sechs (wie in der Formel VIIa) sein kann (s. auch S. 229), liegt hier der Fall vor, daß man aus der Koordinationszahl keinen Sehluß auf die Konstitution ziehen kann. Es bleibt deshalb unentschieden, ob dem sauren Kaliumsalz die Konstitutionsformel Nr. VII oder VII a zukommt.

Die sehr gut kristallisierende Verbindung Nr. I ist frei von Base und enthält auf ein Atom Kobalt zwei Brenzkatechinreste und $51 / 2$ Mol. Wasser. Dem Wassergehalt zufolge könnte sie das saure Brenzkatechinat des Tetraquokobaltokations darstellen:

$$
\left[\mathrm{Co}\left(\mathrm{H}_{2} \mathrm{O}\right)_{4}\right]\left[\mathrm{C}_{6} \mathrm{H}_{4}(\mathrm{OH}) \mathrm{O}\right]_{2} \cdot 1.5 \mathrm{H}_{2} \mathrm{O} \text {. }
$$

Da die Verbindung jedoch (wie die anderen) alkalibeständig ist, kann sie nicht dieses Kation enthalten, sondern sie muß vielmehr wie die bisher besprochenen Salze von einem komplexen Anion abgeleitet werden, und zwar kann es sich nur um das Dibrenzkatechinato-kobalto-anion handeln. Das Kation wird von zwei Wasserstoffatomen gebildet. Man hat es also in dieser Verbindung mit der freien Dibrenzkatechinato-kobaltosäure zu tun, welche allen denjenigen bis jetzt besprochenen Verbindungen zugrunde liegt, die auf ein Kobaltatom zwei Brenzkatechinreste enthalten:

$$
\text { I. }\left[\mathrm{Co}\left({ }_{\mathrm{O}}^{\mathrm{O}} \mathrm{C}_{8} \mathrm{H}_{4}\right)_{2}\right] \mathrm{H}_{2} \cdot 5^{1}{ }_{2} \mathrm{H}_{2} \mathrm{O} \cdot{ }^{1}
$$

Es ist zu bemerken, daß man auch die freie Säure der Dibrenzkatechinato-ferrisalze (S. 223) darstellen kann, während dies bei

1 Über diesen Wassergehalt siehe unten S. 234 u. S. 235. 
den Tribrenzkatechinatosalzen des dreiwertigen Eisens und des zweiwertigen Kobalts, sowie bei den Brenzkatechinato-aluminiumsalzen nicht gelang.

Das Pyridinsalz Nr. VI enthält auf vier Atome Kobalt acht Brenzkatechinreste und $8 \mathrm{Mol}$. Pyridin, aber außerdem noch ein ganzes Mol. Brenzkatechin. Es kann wohl kein Zweifel darüber bestehen, daß anch diese Verbindung ein Salz der Dibrenzkatechinatokobaltosäure vorstellt, und zwar das normale Pyridinsalz. Auffallend ist lediglich, daß auf $4 \mathrm{Mol}$. dieses Salzes noch ein weiteres Mol. Brenzkatechin kommt:

$$
\text { VI. }\left[\mathrm{Co}\left(\mathrm{C}_{6} \mathrm{H}_{4} \mathrm{O}_{2}\right)_{2}\right] \mathrm{H}_{2} \cdot\left(\mathrm{C}_{5} \mathrm{H}_{5} \mathrm{~N}\right)_{2}+\mathrm{C}_{6} \mathrm{H}_{4}(\mathrm{OH})_{2} \text {. }
$$

Derartige Verbindungen des Brenzkatechins mit Salzen der verschiedensten Art wurden auch sonst schon beobachtet. ${ }^{1}$ So . vereinigt sich (1.) das Dikalium-tribrenzkatechinato-aluminiat mit 3 Mol. Brenzkatechin (und 2.5 Mol. Wasser), ferner (2.) das Natriumformiat mit 2 Mol., das Kaliumacetat (3.) und das Kaliumbenzolsulfonat (4.) mit je $1 \mathrm{Mol}$. und das Calciumchlorid (5.) mit 4 Mol. Brenzkatechin (und 2 Mol. Äthylalkohol):

1. $\left[\mathrm{Al}\left(\mathrm{C}_{6} \mathrm{H}_{4} \mathrm{O}_{2}\right)_{3}\right] \mathrm{K}_{2} \mathrm{H} .3 \mathrm{C}_{6} \mathrm{H}_{4}(\mathrm{OH})_{2} \cdot 2.5 \mathrm{H}_{2} \mathrm{O} ; 2 . \mathrm{HCOONa}$. $2 \mathrm{C}_{6} \mathrm{H}_{4}(\mathrm{OH})_{2} ; 3 . \mathrm{CH}_{3} \mathrm{COOK} . \mathrm{C}_{6} \mathrm{H}_{4}(\mathrm{OH})_{2} ; 4 . \mathrm{C}_{6} \mathrm{H}_{5} \mathrm{SO}_{3} \mathrm{~K} . \mathrm{C}_{6} \mathrm{H}_{4}(\mathrm{OH})_{2}$; 5. $\mathrm{CaCl}_{2} \cdot 4 \mathrm{C}_{6} \mathrm{H}_{4}(\mathrm{OH})_{2} \cdot 2 \mathrm{C}_{2} \mathrm{H}_{5} \mathrm{OH}$.

In diesen Fällen verbindet sich das Brenzkatechin in analoger Weise mit dem Metallatom, wie sich das Wasser mit Metallatomen zu Aquokationen vereinigt:
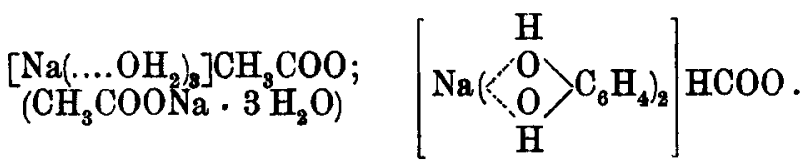

Dies muß auch für das Pyridinsalz der Dibrenzkatechinatokobalto-säure zutreffen: $1 \mathrm{Mol}$. Brenzkatechin vereinigt sich mit einem der 4 Mol. dieses Salzes, und zwar lagert es sich, wie in den obigen Fällen, an die Metallatome, so hier an das Pyridin bzw. an eine Pyridiniumgruppe an:

VI. $3\left[\mathrm{Co}\left(\mathrm{C}_{6} \mathrm{H}_{4} \mathrm{O}_{2}\right)_{2}\right] \mathrm{H}_{2} \cdot\left(\mathrm{C}_{5} \mathrm{H}_{5} \mathrm{~N}\right)_{2} \cdot\left[\mathrm{Co}\left(\mathrm{C}_{6} \mathrm{H}_{4} \mathrm{O}_{2}\right)_{2}\right] \underset{\mathrm{H} \cdot \mathrm{C}_{5} \mathrm{H}_{5} \mathrm{~N}}{\mathrm{H} \cdot \mathrm{C}_{6} \mathrm{H}_{5} \mathrm{~N}}+\mathrm{C}_{6} \mathrm{H}_{4}(\mathrm{OH})_{2}$.

Dieses Salz zeigt, wie das saure Kaliumsalz (S. 230), eine von den übrigen Verbindungen des Dibrenzkatechinato-kobalto-anions ab-

1 Weintand u. W. Denzed, Ber. deutseh. chem. Ges. 47 (1914), 2244, 2990. 
weichende Farbe, nämlich ein helles Fleischrot. Ein Grund hierfür ist nicht ersichtlich; vermutlich ist es das Kation, das diese Farbänderung bewirkt. Daß an sich farblose Ionen die Farbe von Verbindungen mit einem und demselben komplexen gefärbten Ion beeinflussen können, wurde schon bei der Besprechung der Ursachen für die violette Farbe des sauren Kaliumsalzes (S. 231) erwähnt.

Die Ammoniumverbindung Nr. IV enthält auf ein Atom Kobalt drei Brenzkatechinreste, vier Ammoniumgruppen und außerdem noch ein ganzes Mol. Brenzkatechin. Da auf ein Atom Kobalt vier Ammoniumgruppen kommen, $m u B$ dem Salz, wie der an erster Stelle (S. 229) besprochenen Kaliumverbindung Nr. VIII, das vierbasische Tribrenzkatechinato-kobalto-anion zugrunde liegen:

$$
\text { IV. }\left[\mathrm{Co}\left(\mathrm{C}_{6} \mathrm{H}_{4} \mathrm{O}_{2}\right)_{3}\right]\left(\mathrm{NH}_{4}\right)_{4} \cdot \mathrm{C}_{6} \mathrm{H}_{4}(\mathrm{OH})_{2} \text {. }
$$

Das ganze Mol. Brenzkatechin ist, wie im vorhergehenden Pyridinsalz, mit einer der Ammoniumgruppen vereinigt.

Das basenfreie Kobaltbrenzkatechinat Nr. V,

$$
\mathrm{Co}_{3}\left(\mathrm{C}_{6} \mathrm{H}_{4} \mathrm{O}_{2}\right)_{2}(\mathrm{OH})_{2} \cdot 6 \mathrm{H}_{2} \mathrm{O} \text {, }
$$

enthält auf drei Kobaltatome zwei Brenzkatechinreste. Über seine Konstitution läßt sich etwas Sicheres nicht aussagen, da die basischen Salze sehr verschieden konstituiert sein können und man nicht zu entscheiden imstande ist, welehe Möglichkeit hier vorliegt. Es könnte z. B. das Kobaltosalz eines vierbasischen Dihydro-dibrenzkatechinatö-anions vorstellen (V.) oder ein Tetraquo-kobalto-brenzkatechinat sein, dessen 4 Wassermol. durch 2 Mol. Kobaltohydroxyd ersetzt sind (Va.):

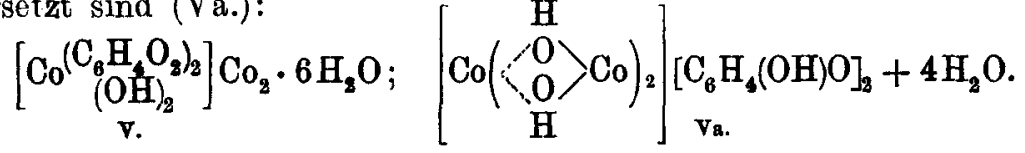

Da die Mehrzahl der obigen Verbindungen wasserhaltig ist, bleibt noch übrig, kurz auf die Art der Bindung des Wassers einzugehen.

Beim violetten Kaliumsalz Nr. VII ist es, wie oben (S. 231) schon erörtert wurde, möglich, daß die beiden Wassermoleküle zum komplexen Anion gehören.

Bei allen anderen Verbindungen sind die Wassermol€küle dagegen zum Kation zu rechnen, man hat es bei diesen mit Aquokationen zu tun. Als Beispiel nennen wir das rote Kaliumsalz Nr. VIII, in welchem vier Monaquo-kalium-kationen enthalten sind:

$$
\text { VIII. } \left.\left[\mathrm{Co}\left(\mathrm{C}_{6} \mathrm{H}_{4} \mathrm{O}_{2}\right)_{8}\right] \mathrm{K} \ldots \mathrm{OH}_{2}\right]_{4} \text {. }
$$


Die freie Dibrenzkatechinato-kobaltosäure Nr. I (S. 224 u. 231) enthält 51/2 Mol. Wasser. ${ }^{1}$ Daß derartige komplexe Säuren wasserhaltig sind, ist eine allgemeine Beobachtung. So enthält z. B. die Hexachloro-platt säure $6 \mathrm{Mol}$. Wasser, die Tetrachloro-aurisäure $4 \mathrm{Mol}$. und die violette Dibrenzkatechinato-ferrisäure (S. 223) 2 Mol. Wasser. Man nimmt bei ihnen das Wasser mit den Wasserstoffatomen zu Aquokationen verbunden an. Demgemäß befinden sich in $2 \mathrm{Mol}$. der Dibrenzkatechinato-kobaltosäure drei Triaquo-wasserstoffkationen und ein Diaquo-wasserstoffkation:

I. $\left.\left[\mathrm{Co}\left(\mathrm{C}_{6} \mathrm{H}_{4} \mathrm{O}_{2}\right)_{2}\right]_{2}\left[\mathrm{H} \ldots . . .\left(\mathrm{OH}_{2}\right)_{2}\right)_{3}\right]$.

\section{Versuchsteil.}

I. Dibrenzkatechinato-kobaltosäure.

(Vergleiche oben S. 231 u. 235.)

Wie im allgemeinen Teil, S. 235, schon ausgetührt wurde, erhält man diese Säure aus Lösungen von 1 Mol. Kobaltosalz, 3 Mol. Brenzkatechin und 2 bis 3 Mol Ammoniak. Hierbei scheidet sich auf den Zusatz des Ammoniaks zunächst die pulverförmige Verbindung $\mathrm{Nr} . \mathrm{V}$ aus. Diese verwandelt sich aber in der Fällungsflüssigkeit, je nach den Umständen (siehe unter Nr. V, S. 237) mehr oder weniger vollständig in die in Rede stehende Säure.

$\mathrm{Zu}$ ihrer Darstellung verfährt man folgendermaßen: Man löst $2.8 \mathrm{~g}$ Kobaltsulfat und $3.3 \mathrm{~g}$ Brenzkatechin unter Erwärmen in $30 \mathrm{ccm}$ Wasser, läßt erkalten und fügt unter Umrühren $21 / 2 \mathrm{~cm} 15 \%$ iges Ammoniak hinzu (1 Co: $3 \mathrm{C}_{6} \mathrm{H}_{4}(\mathrm{OH})_{2}$ : $2 \mathrm{NH}_{3}$ ). Der zunächst ausfallende pulverförmige Niederschlag (Nr. V) verwandelt sich innerhalb weniger Stunden vollständig in die kristallinische Säure. Man saugt ab, wäscht mit kaltem Wasser und trocknet über Chlorcalcium.

Hellrote, vier- oder sechsseitige, schief abgeschnittene Säulchen. Die Verbindung behält ihre rote Farbe verhältnismäBig lang, die oberflächliche Dunkelfärbung durch Oxydation (s. o. S. 227) findet langsam statt. In Wasser ist die Verbindung schwerlöslich.

$$
\text { Analyse. }
$$

Zur Bestimmung des Kobalts wurden die Verbindungen verascht, der Rückstand in Salzsäure unter längerem Erwärmen auf dem Wasserbad gelöst, die Salzsäure durch Abrauchen mit Schwefelsäure vertrieben und das Kobalt aus stark ammoniakalischer Lösung elektrisch abgeschieden.

$0.5264 \mathrm{~g}$ Sbst.: $0.0830 \mathrm{~g}$ Co. $-0.6232 \mathrm{~g}$ Sbst.: $0.1009 \mathrm{~g}$ Co. $-0.5126 \mathrm{~g}$ Sbst.: $0.0821 \mathrm{~g}$ Co. $-0.5234 \mathrm{~g}$ Sbst.: $0.0852 \mathrm{~g}$ Co. $-0.2586 \mathrm{~g}$ Sbst.: $0.3644 \mathrm{~g}$ $\mathrm{CO}_{2}, 0.1300 \mathrm{~g} \mathrm{H}_{2} \mathrm{O}$. $-0.1896 \mathrm{~g}$ Sbst.: $0.2665 \mathrm{~g} \mathrm{CO}_{2}, 0.0959 \mathrm{~g} \mathrm{H}_{2} \mathrm{O}-0.1879 \mathrm{~g}$ Sbst.: $0.2651 \mathrm{~g} \mathrm{CO}_{2}, 0.0908 \mathrm{~g} \mathrm{H}_{2} \mathrm{O}$. $-0.1975 \mathrm{~g}$ Sbst.: $0.2773 \mathrm{~g} \mathrm{CO}_{2}, 0.0985 \mathrm{~g}$ $\mathrm{H}_{2}$ O. $-0.1980 \mathrm{~g}$ Sbst. : $0.2806 \mathrm{~g} \mathrm{CO}_{2}, 0.0958 \mathrm{~g} \mathrm{H}_{2} \mathrm{O}$. $-0.2172 \mathrm{~g}$ Sbst.: $0.3049 \mathrm{~g}$ $\mathrm{CO}_{2}, 0.1070 \mathrm{~g} \mathrm{H}_{2} \mathrm{O}$.

1 Über diesen Wassergehalt siehe auch unten S. 235. 


\begin{tabular}{|c|c|c|c|}
\hline \multicolumn{4}{|c|}{$\left[\mathrm{Co}\left(\mathrm{C}_{6} \mathrm{H}_{4} \mathrm{O}_{2}\right)_{2}\right] \mathrm{H}_{2} .5^{1} / 2 \mathrm{H}_{2} \mathrm{O}(376.1)$} \\
\hline Ber. & Co $\mathbf{1 5 . 6 8}$ & C 38.28 & H 5.63 \\
\hline & Co 15.8 & C 38.4 & H 5.6 \\
\hline & Co 16.2 & C 38 . & H 5.7 \\
\hline & Co 16.0 & C 38.5 & H 5.4 \\
\hline & Co 16.3 & C & H 5.6 \\
\hline & & & 4 \\
\hline & & & \\
\hline
\end{tabular}

Es könnte auffallen, daß die Verbindung $5 \frac{1}{2}$ Mol. Wasser enthält und nicht etwa 5 oder 6. Aber sämtliche untereinander gut übereinstimmenden analytischen Werte entsprechen nur diesem Wassergehalte; denn für $5 \mathrm{Mol}$. Wasser sind berechnet: $16.0 \% \mathrm{Co}, 39.2 \% \mathrm{C}, 5.5 \% \mathrm{H}_{2}$ und für $6 \mathrm{Mol}$. Wasser: $15.3 \% \mathrm{Co}$, $37.4 \%$ C, $5.75 \% \mathrm{H}_{2}$.

\section{Ammonium-dibrenzkatechinato-kobaltoat.}

(Vergleiche oben S. 225 u. 229.)

Um dieses Salz einheitlich zu erhalten, muß man sich genau nach den folgenden Angaben richten: Man löst4 $\mathrm{g}$ Kobalt-Ammoniumsulfat $\left(\mathrm{Co}\left(\mathrm{NH}_{4}\right)_{2}\left(\mathrm{SO}_{4}\right)_{2} \cdot 6 \mathrm{H}_{2} \mathrm{O}\right)$ unter Erwärmen in $40 \mathrm{~g}$ Wasser und fügt $2.8 \mathrm{~g}$ Brenzkatechin dazu. Nach dem Erkalten versetzt man die Lösung unter Umrühren mit $10 \mathrm{~cm} 15 \%$ igem Am. moniak ( $1 \mathrm{Co}: 2.5 \mathrm{C}_{6} \mathrm{H}_{4}(\mathrm{OH})_{2}: 9 \mathrm{NH}_{3}$ ). Beim Eingießen des Ammoniaks fält zunächst ein hellrosagefärbter, pulverförmiger Niederschlag aus (siehe unter Nr. V, S.237), der sich jedoch beim Zusatz des übrigen Ammoniaks wieder löst. Die Lösung färbt sich dabei tief dunkelrot. Bald scheidet sich das Salz Nr. II in roten Kriställchen ab, die in Wasser schwer löslich sind und sich daher gut waschen lassen. Man trocknet es über Chlorcalcium.

Sehr schöne, schlanke, vierseitige, gerade abgeschnittene, rote Säulchen. Das Salz ist in der Mutterlauge ganz beständig, dagegen färbt es sich, sobald es gewaschen ist, ziemlich rasch dunkel. Doch beeinträchtigte diese Dunkelfärbung das Ergebnis der Analyse nicht merklich.

$$
\begin{aligned}
& \text { Analyse. } \\
& 0.4727 \mathrm{~g} \text { Sbst.: } 0.0908 \mathrm{~g} \text { Co. }-0.6018 \mathrm{~g} \text { Sbst.: } 0.1158 \mathrm{~g} \text { Co. }-0.3579 \mathrm{~g}
\end{aligned}
$$
Sbst.: $22.59 \mathrm{ccm} 1 / 10^{-n}$. HCl. $-0.2894 \mathrm{~g}$ Sbst.: $18.49 \mathrm{ccm} 1 / 10^{-n}$. HCl. -

\begin{tabular}{|c|c|c|c|c|}
\hline \multicolumn{5}{|c|}{$\left[\mathrm{Co}\left(\mathrm{C}_{6} \mathrm{H}_{4} \mathrm{O}_{2}\right)_{2}\right]\left(\mathrm{NH}_{4}\right)_{2}(311.1)}$. \\
\hline $\begin{array}{l}\text { Ber. } \\
\text { Gef. }\end{array}$ & $\begin{array}{l}\text { Co } 18.96 \\
\text { Co } 19.21 \\
\text { Co } 19.24\end{array}$ & $\begin{array}{ll}\mathrm{NH}_{3} & 10.95 \\
\mathrm{NH}_{3} & 10.76 \\
\mathrm{NH}_{8} & 10.88\end{array}$ & $\begin{array}{l}\text { C } 46.3 \\
\text { C } 46.0\end{array}$ & $\begin{array}{ll}\text { H } & 5.18 \\
\text { H } & 4.9\end{array}$ \\
\hline
\end{tabular}
$0.2064 \mathrm{~g}$ Sbst.: $0.3480 \mathrm{~g} \mathrm{Co}_{2}, 0.0906 \mathrm{~g} \mathrm{H}_{2} \mathrm{O}$.

III. Saures Ammonium-dibrenzkatechinato-kobaltoat.

(Vergleiche oben S. 325 u. 230.)

Bei der Darstellung dieser Verbindung muß man sich genau an die folgenden Angaben halten und zwar sowohl bezüglich der Mengenverhältnisse und der Konzentration als auch bezüglich der Trennung des Salzes von der Mutterlauge.

$2.8 \mathrm{~g}$ Kobaltosulfat und $3.8 \mathrm{~g}$ Brenzkatechin werden unter Erwärmen in. $25 \mathrm{ccm}$ Wasser gelöst. Nach dem Erkalten fügt man $6 \mathrm{ccm} 15 \%$ iges Ammoniak 
hinzu (1 Co : $3.5 \mathrm{C}_{6} \mathrm{H}_{4}(\mathrm{OH})_{2}: 5 \mathrm{NH}_{3}$ ). Es entsteht sofort der rötliche, pulverförmige Niederschlag des Salzes Nr. V. Dieser verwandelt sich aber bald in das eisblumenartig kristallisierte, in der Uberschrift genannte Salz. Man wäsoht es nach 12 Stunden dekantierend, streicht es auf Ton, trocknet kurze Zeit und füllt es in Gläschen.

Das Salz bildet hellrote, eisblumenartig aggregierte Wachstumsanfänge, die sich, selbst wenn sie sich wochenlang in der Mutterlauge befinden, nicht verändern. In Wasser ist es ziemlich schwer löslich.

Wird das Salz nach dem Waschen auf der Nutsche abgesaugt, so ist es sehr unbeständig und zersetzt sich, indem es im Gläschen feucht wird.

Analyoe.

$0.4291 \mathrm{~g}$ Sbst.: $0.0826 \mathrm{~g}$ Co. $-0.4505 \mathrm{~g}$ Sbst.: $0.0879 \mathrm{~g}$ Co. $-0.5443 \mathrm{~g}$ Sbst.: $21.68 \mathrm{ccm} 1 / 10^{-n}$. HCl. - $0.4883 \mathrm{~g}$ Sbst.: $19.15 \mathrm{ccm} 1 / 10^{-\mathrm{n}}$. HCl. $-0.1596 \mathrm{~g}$ Sbst.: $0.2800 \mathrm{~g} \mathrm{CO}_{2}, 0.0669 \mathrm{~g} \mathrm{H}_{2} \mathrm{O}$. $-0.1590 \mathrm{~g}$ Sbst. : $0.2760 \mathrm{~g} \mathrm{CO}_{2}, 0.0648 \mathrm{~g} \mathrm{H}_{2} \mathrm{O}$.

$3\left[\mathrm{Co}\left(\mathrm{C}_{6} \mathrm{H}_{4} \mathrm{O}_{2}\right)_{2}\right]\left(\mathrm{NH}_{4}\right) \mathrm{H}+\left[\mathrm{Co}\left(\mathrm{C}_{6} \mathrm{H}_{4} \mathrm{O}_{2}\right)_{2}\right]\left(\mathrm{NH}_{4}\right)_{2}+\mathrm{H}_{2} \mathrm{O}$ (1211.4).

$\begin{array}{llll}\text { Ber.: } \mathrm{Co} 19.47 & \mathrm{NH}_{3} 7.00 & \mathrm{C} 47.55 & \mathrm{H} 4.74 \\ \text { Gef.: Co } 19.25 & \mathrm{NH}_{3} 6.8 & \mathrm{C} 47.8 & \mathrm{H} 4.7 \\ \text { Co } 19.50 & \mathrm{NH}_{3} 6.7 & \mathrm{C} 47.3 & \mathrm{H} 4.6\end{array}$

IV. Ammonium-tribrenzkatechinato-kobaltoat.

(Vergleiche oben S. 225 u. 233.)

Das Allgemeine über die Bildungsbedingungen dieses Salzes wurde bereits oben $\$ .225$ mitgeteilt. Zu seiner Darstellung verfährt man nach a); nach b) erhält man es in sehr zersetzlichem Zustand.

a) $4 \mathrm{~g}$ Kobaltammoniumsulfat werden unter Erwärmen in $12 \mathrm{cem}$ Wasser gelöst. Nach dem Erkalten fügt man unter vorsichtigem Rühren (siehe unten)

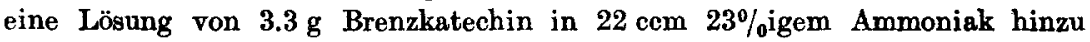
$\left(1 \mathrm{Co}: 3 \mathrm{C}_{6} \mathrm{H}_{4}(\mathrm{OH})_{2}: 27 \mathrm{NH}_{3}\right)$. Bei niedriger Temperatur $\left(0^{\circ}\right)$ scheidet sich über Nacht das fragliche Salz in ziemlich großen, dünnen, schwach rosa geförbten Blättchen aus, die einige Mal mit eiskaltem Wasser gewaschen, abgesaugt, auf Ton gestrichen und über Natronkalk getrocknet werden. Rührt man beim Hinzugießen des Ammoniaks zu heftig, so fällt der Körper sofort aus, und zwar in äußerst dünnen, stark lichtbrechenden Blättchen, die, nachdem sie von der Mutterlauge getrennt und ausgewaschen werden, sich sehr rasch schwarz färben und nach Ammoniak riechen. Die bei obiger Darstellungsweise entstehenden dickeren Kriställchen sind dagegen beständiger.

b) Das Verhältnis der angewandten Mengen Kobaltosalz, Brenzkatechin und Ammoniak ist dasselbe wie bei der Darstellung des Ammonium-dibrenzkatechinato-kobaltosalzes Nr. II, S. 235, aber man arbeitet in konzentrierterer Lösung (1 Co: $\left.2.5 \mathrm{C}_{6} \mathrm{H}_{4}(\mathrm{OH})_{2}: 9 \mathrm{NH}_{3}\right): 4 \mathrm{~g}$ Kobaltammoniumsulfat werden unter Erwärmen in $18 \mathrm{ccm}$ Wasser gelöst. In dieser Lösung löst man $2.8 \mathrm{~g}$ Brenzkatechin und versetzt sie nach dem Erkalten mit $71 / \mathrm{ccm} \mathrm{23 \%}$ igem Ammoniak. Dabei entsteht sofort eine große Menge eines Niederschlags, der aus äußerst dünnen, stark lichtbrechenden, farblos scheinenden Blättchen besteht. Fr muß sofort von der Mutterlauge getrennt werden, denn nach kurzer Zeit findet man ihn vermischt mit den schönen, roten Säulchen der Verbindung Nr. I, S. 235. 
Aber wie schon bei a) bemerkt, sind die so erhaltenen dünnen Blättchen sehr zersetzlich und zur Analyse nicht geeignet. Arbeitet man in der Hitze, so erhält man die dünnen Blättchen überhaupt nicht allein, sondern sogleich ein Gemenge der Verbindungen Nr. IV und II, in dem die roten Säulchen des Salzes Nr. II vorwiegen.

Das Salz bildet dünne, bald schmälere, bald breitere, länglich $\Theta$ Täfelchen, die teils gerade, teils schief abgeschnitten, teils dachförmig begrenzt sind. Wenn die Täfelchen sehr dünn sind, erscheinen sie farblos, die etwas dickeren sind blaßrosa gefärbt. In Wasser ist das Salz sehr leicht löslich. Sobald es isoliert ist, färbt es sich dunkel.

Nach a) dargestellt:

Analyge.

$0.4023 \mathrm{~g}$ Sbst. : $0.0432 \mathrm{~g}$ Co. $-0.4842 \mathrm{~g}$ Sbst.: $0.0506 \mathrm{~g}$ Co. $-0.8506 \mathrm{~g}$ Sbst.: $0.0884 \mathrm{~g}$ Co. $-0.2358 \mathrm{~g}$ Sbst.: $16.16 \mathrm{~cm} 1 / 10^{-\mathrm{n}}$. HCl. $-0.2991 \mathrm{~g}$ Sbst.: $20.15 \mathrm{ccm} \mathrm{1/10}$-n. HCl. $-0.2060 \mathrm{~g}$ Sbst.: $0.3841 \mathrm{~g} \mathrm{CO}_{2}, 0.1114 \mathrm{~g} \mathrm{H}_{2} \mathrm{O}$.

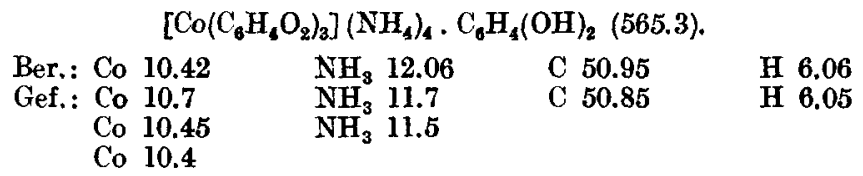

\section{Basisches Kobaltbrenzkatechinat.}

(Vergleiche oben S. 225 u. 233.)

Dieses basische Salz fällt, wie oben \$. 234 schon mitgeteilt wurde, bei der Darstellung der Dibrenzkatechinato-kobaltosäure auf Zusatz des Ammoniaks zunächst als pulveriger Niederschlag aus. Es erleidet aber in jener Mutterlauge rasch die Umwandlung in die Säure (vergleiche außerdem noch das vorübergehende Auftreten des Salzes bei der Darstellung der Ammoniumsalze II u. III). Um diese Umwandlung in die Säure sehr zu verlangsamen, muß bei höherer Temperatur und in verdünnterer Lösung gearbeitet werden und außerdem muß die Menge des angewandten Brenzkatechins vermindert werden.

a) $2.8 \mathrm{~g}$ Kobaltsulfat werden unter Erwärmen in $40 \mathrm{ccm}$ Wasser gelöst und $2.2 \mathrm{~g}$ Brenzkatechin dazugefügt. $\mathrm{Zu}$ der etwa $50^{\circ}$ warmen Lösung gibt man 2\% ccm 15\% iges Ammoniak ( $1 \mathrm{Co}_{2}: 2 \mathrm{C}_{6} \mathrm{H}_{4}(\mathrm{OH})_{2}: 2 \mathrm{NH}_{3}$ ). Das basische Brenzkatechinat scheidet sich wiederum sogleich aus. In dieser Mutterlauge findet nun die Umwandlung in die Säure nur sehr langsam statt. Erst nach mehreren Tagen beobachtet man in dem blaßroten, pulverförmigen Niederschlag einzelne der charakteristischen, schief abgeschnittenen Säulchen der Säure. Man saugt den Niederschlag nach etwa 12 Stunden ab, wäscht ihn mit kaltem Wasser, streicht ihn auf Ton und trocknet über Chlorcalcium.

b) Man bekommt dieses basische Salz aber auch aus einer Lösung, die mehr Brenckatechin enthìlt, wenn man noch verdünnter arbeitet: Man löst $2.8 \mathrm{~g}$ Kobaltsulfat, $3.3 \mathrm{~g}$ Brenzkatechin und $60 \mathrm{ccm}$ Wasser und fügt $21 / \mathrm{ccm}$ $16 \%$ iges Ammoniak rinzu $\left(1 \mathrm{Co}: 3 \mathrm{C}_{6} \mathrm{H}_{4}(\mathrm{OH})_{2}: 2 \mathrm{NH}_{3}\right)$. 
Das Salz bildet, frisch dargestellt, ein blaßrotes Pulver, färbt sich aber an der Luft rasch dunkelgrün. Es ist in Wasser schwer löslich.

$$
\text { Analyse. }
$$

a) $0.5817 \mathrm{~g}$ Sbst.: $0.1922 \mathrm{~g}$ Co. $-0.2076 \mathrm{~g}$ Sbst.: $0.2147 \mathrm{~g} \mathrm{CO}_{2}, 0.0612 \mathrm{~g}$

b) $0.2995 \mathrm{~g}$ Sbst. : $0.1046 \mathrm{~g}$ Co. $-0.1858 \mathrm{~g}$ Sbst.: $0.1892 \mathrm{~g} \mathrm{CO}_{2}, 0.0498 \mathrm{~g} \mathrm{H}_{2} \mathrm{O}$.

\begin{tabular}{llll}
\multicolumn{4}{c}{$\mathrm{Co}_{3}\left(\mathrm{C}_{6} \mathrm{H}_{4} \mathrm{O}_{2}\right)_{2}(\mathrm{OH})_{2} .6 \mathrm{H}_{2} \mathrm{O}$ (535.1). } \\
Ber.: & $\mathrm{Co} 33.06$ & C 27.54 & $\mathrm{H} 4.14$ \\
Gef.: a; $\mathrm{Co} 33.0^{1}$ & C 28.2 & $\mathrm{H} 3.3$ \\
b) Co $34.9^{1}$ & C 27.7 & H 3.0
\end{tabular}

VI. Pyridin-dibrenzkatechingto-kobaltoat.

(Vergleiche oben S. 225 u. 232.)

Während man aus Kobaltosalzlösungen. Brenzkatechin und Ammoniak je nach den Mengenverhältnissen und der Konzentration die im Vorhergehenden beschriebenen fünf Verbindungen ( $\mathrm{I}-\mathrm{V}$ ) erhalten kann, bekommt man bei Verwendung von Pyrioin an Stelle des Ammoniaks, wie schon oben S.225 hervorgehoben wurde, nur das in der Überschrift genannte Salz. Zu seiner Darstellung löst man $1.4 \mathrm{~g}$ Kobaltsulfat und $1.4 \mathrm{~g}$ Brenzkatechin in $50 \mathrm{ccm}$ Wasser und fügt $1.6 \mathrm{ccm}$ Pyridin, verdünnt mit $50 \mathrm{ccm}$ Wasser, hinzu $\left(1 \mathrm{Co}: 2.5 \mathrm{C}_{6} \mathrm{H}_{4}(\mathrm{OH})_{2}\right.$ : $4 \mathrm{C}_{5} \mathrm{H}_{6} \mathrm{~N}$ ). Das Salz fällt sofort als pulverförmiger, fleischfarbener Niederschlag aus. Nach 12 Stunden wird es abgesaugt, mit kaltem Wasser gewaschen, auf Ton gestrichen und über Natronkalk getrocknet.

Das Salz bildet ein fleischrotes Pulver, das sich unter dem Mikroskop als kugelförmige Aggregate von feinen, nadelförmigen Kriställchen erweist. Die Versuche, das Salz in größeren Kristallen zu erhalten, waren ohne Erfolg. Im Gegensatz zu den anderen Verbindungen ist dieses Pyridinsalz an der Luft beständig, es verändert seine Farbe nicht oder nur sehr wenig. In Wasser ist es schwer löslich.

$$
\text { Analyse. }
$$

$0.5557 \mathrm{~g}$ Sbst.: $0.0707 \mathrm{~g}$ Co. $-0.5587 \mathrm{~g}$ Sbst.: $0.0711 \mathrm{~g}$ Co. $-0.5437 \mathrm{~g}$ Sbst.: $23.97 \mathrm{ccm} 1 / 10^{-n}$. HCl. ${ }^{2}-0.4357 \mathrm{~g}$ Sbst.: $19.18 \mathrm{ccm} 1 / 10^{-n} . \mathrm{HCl}^{2}$. $0.1279 \mathrm{~g}$ Sbst.: $0.2882 \mathrm{~g} \mathrm{CO}_{2}, 0.0570 \mathrm{~g} \mathrm{H}_{2} \mathrm{O}$. $0.1220 \mathrm{~g}$ Sbst.: $0.2748 \mathrm{~g} \mathrm{CO}_{2}$, $0.0549 \mathrm{~g} \mathrm{H}_{2} \mathrm{O}$.

1 Die Übereinstimmung der Kobaltwerte der nach den beiden Methoden a) und b) dargestellten Proben der Verbiudung ist nicht ganz befriedigend. Indessen kann bei einer pulverförmigen, sich an der Luft stark dunkel färbenden Substanz, deren Einheitlichkeit erst aus der Analyse hervorgeht, nicht mehr erwartet werden. Mit der oberflächlichen Oxydation hängt wohl auch der zu niedrige Wert für Wasserstoff zusammen.

2 Pyridinbestimmung durch Titration mit Methylorange als Indikator unter Verwendung einer Vergleichslösung, siehe Z. anorg. Chem. 80 (1913), 428. 


\begin{tabular}{|c|c|c|c|}
\hline \multicolumn{4}{|c|}{$4\left[\mathrm{Co}\left(\mathrm{C}_{6} \mathrm{H}_{4} \mathrm{O}_{2}\right)_{2}\right] \mathrm{H}_{2} \cdot\left(\mathrm{C}_{5} \mathrm{H}_{5} \mathrm{~N}\right)_{2} \cdot \mathrm{C}_{6} \mathrm{H}_{4}(\mathrm{OH})_{2}(1850.6)$} \\
\hline Co 12.74 & Pyr. 34.17 & C 60.95 & H 4.68 \\
\hline Gef.: Co 12.73 & Pyr. 34.85 & C $61.45^{1}$ & H $4.98^{1}$ \\
\hline Co 12.72 & 34.82 & C $61.43^{1}$ & H $5.03^{1}$ \\
\hline
\end{tabular}

VII. Saures Kalium-dibrenzkatechinato-kobaltoat.

(Vergleiche oben S. 226 u. 230.)

Zur Darstellung löst man $2.5 \mathrm{~g}$ Kobaltacetat $\left(\mathrm{Co}\left(\mathrm{CH}_{3} \mathrm{COO}\right)_{2} .4 \mathrm{H}_{2} \mathrm{O}\right)$ unter Erwärmen in $10 \mathrm{ccm}$ Wasser und in dieser Lösung $3.3 \mathrm{~g}$ Brenzkatechin. ${ }^{2}$ Nach dem Erkalten der Lösung fügt man unter vorsichtigem Umrühren eine abgekühlte Lösung von $2.25 \mathrm{~g}$ Kaliumhydroxyd in $5 \mathrm{ccm}$ Wasser hinzu (1Co: $\left.3 \mathrm{C}_{8} \mathrm{H}_{4}(\mathrm{OH})_{2}: 4 \mathrm{KOH}\right)$. Dabei fällt zunächst ein hellroter, flockiger Niederschlag $^{3}$ aus, der sich innerhalb einiger Stunden in die violetten Kriställchen des in Rede stehenden Kaliumsalzes verwandelt. Man wäscht es einige MaJ dekantierend mit eiskaltem Wasser, streicht es auf Ton und trocknet über Chlorcalcium.

Das Salz bildet rot- bis blauviolette, dünne, längliche, meist schmale Täfelchen. Diese sind in der Regel gerade abgeschnitten, zum Teil zeigen sie auch eine abgestumpfte Ecke. 'Sie bilden häufig Kristallaggregate. Jas Salz löst sich leicht in Wasser mit schmutzig grüner Farbe.

\section{Analyse.}

Zur Bastimmung der Alkalien wurden die Salze mit konzentrierter Schwefelsäure vorsichtig abgeraucht bis zur völligen Zersetzung des Brenzkatechins. Hierauf wurde das Kobalt mit farblosem Schwefelammon abgeschieden.

$0.3681 \mathrm{~g}$ Sbst.: $0.0616 \mathrm{~g}$ Co. $-0.5629 \mathrm{~g}$ Sbst.: $0.0956 \mathrm{~g}$ Co. $-0.6713 \mathrm{~g}$ Sbst.: $0.1652 \mathrm{~g} \mathrm{~K}_{2} \mathrm{SO}_{4}$. $-0.6057 \mathrm{~g}$ Sbst.: $0.1522 \mathrm{~g} \mathrm{~K}_{2} \mathrm{SO}_{4}$. $-0.1909 \mathrm{~g}$ Sbst.: $0.2917 \mathrm{~g} \mathrm{CO}_{2}$.

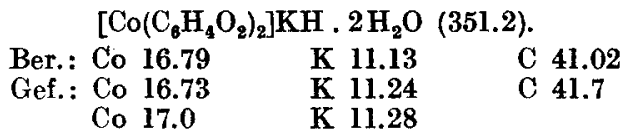

VIII. Kalium-tribrenzkatechinato-kobaltoat.

(Vergleiche oben S. $226 \mathrm{u}$ u. 228.)

Man löst $1.25 \mathrm{~g}$ Kobaltacetat unter Erwärmen in $4 \mathrm{ccm}$ Wasser, fügt zu der warmen Lösung $1.65 \mathrm{~g}$ Brenzkatechin und die heiße Lösung von $11.2 \mathrm{~g}$ Kaliumhydroxyc in $8 \mathrm{ccm}$ Wasser $\left(1 \mathrm{Co}: 3 \mathrm{C}_{8} \mathrm{H}_{4}(\mathrm{OH})_{2}: 40 \mathrm{KOH}\right)$.

1 Der etwas zu hohe Gehalt an Kohlenstoff und Wasserstoff rührt wohl daher, daß das verwandte Pyridin'homologe Basen, wie Picolin, in geringer Menge enthielt, deren Dibrenzkatechinato-kobaltosalze das Pyridinsalz verunreinigen.

2 Erwärmt man eine wässerige Lösung von Kobaltacetat una Brenzkatechin, so scheidet sich ein Kobaltbrenzkatechinat in rötlicher, kristallinischer Kruste aus, das wir his jetzt nicht näher untersucht haben. Man kann daher das Brenzkatechin nicht zugleich mit dem Kobaltacetat unter Erwärmen auflösen.

${ }^{3}$ Dieser wurde bis jetzt nicht untersucht. 
Beim Eingieben der Kalilauge bildet sich zunächst ein hellroter, flockjger Niederschlag, der sich jedoch beim Zusatz der übrigen Kalilauge wieder löst. Die Lösung ist schließlich tiefblau und behält diese Farbe auch nach dem Erkalten. Beim Abkühlen der Lösung scheiden sich rasch feine, nadelförmige, scheinbar farblose Kriställchen aus, die sich in der Mutterlauge in wenigen Stunden zu roten, schlanken, vierseitigen, gerade abgeschnittenen Säulchen vergrößern. Diese stellen das in Rede stehende Salz dar. Man wïscht es einmal mit eiskaltem Wasser, streicht es auf Ton und trocknet über Chlorcalcium, wobei es seine schöne rote Farbe bald verliert und sich dunkel färbt.

Das Salz ist in Wasser sehr leicht löslich mit roter Farbe.

Analyse.

$0.3900 \mathrm{~g}$ Sbst.: $0.0365 \mathrm{~g}$ Co. $-0.8709 \mathrm{~g}$ Sbst.: $0.0809 \mathrm{~g}$ Co. $-0.6668 \mathrm{~g}$ Sbst.: $0.3870 \mathrm{~g} \mathrm{~K}_{2} \mathrm{SO}_{4}$. $-0.1287 \mathrm{~g}$ Sbst.: $0.1608 \mathrm{~g} \mathrm{CO}_{2}, 0.0361 \mathrm{~g} \mathrm{H}_{2} \mathrm{O}$.

$$
\begin{aligned}
& {\left[\mathrm{Co}\left(\mathrm{C}_{6} \mathrm{H}_{4} \mathrm{O}_{2}\right)_{3}\right] \mathrm{K}_{4} \text {. } 4 \mathrm{H}_{2} \mathrm{O}(611.5) \text {. }} \\
& \text { Ber.: Co } 9.64 \quad \text { IK } 25.58 \quad \text { C } 35.32 \quad \text { H } 3.29 \\
& \text { Gef.: Co } 9.4 \quad \text { K } 26.0 \quad \text { C } 34.1 \quad \text { H } 3.1 \\
& \text { Co } 9.3
\end{aligned}
$$

Aus den für Kobalt und Kalium gefundenen Werten ergibt sich das Verhältnis Kobalt: Kalium $=1: 4.2$. Hieraus geht hervor, daB die analysierte Probe durch angetrocknete Mutterlauge $0.2 \mathrm{Mol}$. KOH zu viel enthielt. Berechnet man hiernach die Prozente, so ergeben sich: 9.47\% Co, 26.37\% K, $34.69 \% \mathrm{C}, 3.27 \% \mathrm{H}$, womit die gefundenen Werte gut übereinstimmen.

Tübingen, Chemisches Laboratorium der Universität, 18. Nov. 1917

Bei der Redaktion eingegangen am 21. November 1917. 